\title{
Sorting chromatic sextupoles for easily and effectively correcting second order chromaticity in the Relativistic Heavy Ion Collider
}

\author{
Y. Luo, S. Tepikian, W. Fischer, G. Robert-Demolaize, D. Trbojevic
}

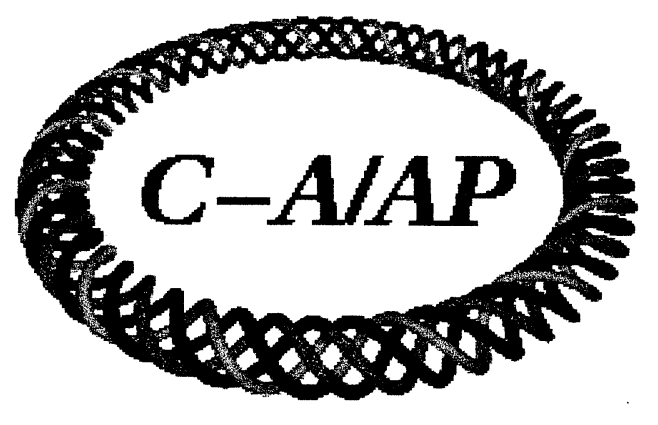

\section{Collider-Accelerator Department Brookhaven National Laboratory Upton, NY 11973}

Notice: This document has been authorized by employees of Brookhaven Science Associates, LLC under Contract No. DE-AC02-98CH10886 with the U.S. Department of Energy. The United States Government retains a nonexclusive, paid-up, irrevocable, world-wide license to publish or reproduce the published form of this document, or allow others to do so, for United States Government purposes. 


\title{
Sorting chromatic sextupoles for easily and effectively correcting second order chromaticity in the Relativistic Heavy Ion Collider
}

\author{
Y. Luo, S. Tepikian, W. Fischer, G. Robert-Demolaize, D. Trbojevic \\ Brookhaven National Laboratory, Upton, NY 11973, USA
}

Based on the contributions of the chromatic sextupole families to the half-integer resonance driving terms, we discuss how to sort the chromatic sextupoles in the arcs of the Relativistic Heavy Ion Collider (RHIC) to easily and effectively correct the second order chromaticities. We propose a method with 4 knobs corresponding to 4 pairs of chromatic sextupole families to online correct the second order chromaticities. Numerical simulation justifies this method, showing that this method reduces the unbalance in the correction strengths of sextupole families and avoids the reversal of sextupole polarities. Therefore, this method yields larger dynamic apertures for the proposed RHIC 2009 100GeV polarized proton run lattices.

\section{Introduction}

There are 144 chromatic sextupoles in the 6 arcs in each ring of the Relativistic Heavy Ion Collider (RHIC). Before 2007, there were 12 power supplies for them in each ring. In each arc there are two independent power supplies, one for all focusing sextupoles (SFs) and one for all defocusing sextupoles (SDs). In the RHIC operation before 2007, a 2-family chromaticity correction scheme was used. The 2-family correction scheme can only correct the first order chromaticities.

During the Summer shutdown of RHIC 2006, the number of the power supplies for the arc chromatic sextupoles were doubled from 12 to 24 to allow the correction of the second order chromaticities. For the RHIC polarized proton (pp) runs, the second order chromaticity correction turns out to be important with the increased bunch intensity and reduced $\beta^{*}$ at the interaction points (IPs) [1].

After doubling the power supplies, in each arc there now are power supplies for the SF sextupoles and two for the SD sextupoles. The SF and SD sextupoles in each arc are split into two SF and two SD sub-families. The chromatic sextupoles in the 3 outer arcs are sorted as $6 *$ (SFPO, SDMO, SFMO, SDMO), while the chromatic sextupoles in the 3 inner arcs are sorted as $6^{*}$ (SFPI, SDMI, SFMI, SDMI) [2]. For each sextupole family of SFPO, SDMO, SFMO, SDMO, SFPI, SDMI, SFMI, SDMI, there actually are three independent power supplies, although they are always powered with same strengths in the RHIC control system.

In this article, we first review the periodic optics parameters at all chromatic sextupoles in the RHIC rings. Then we show that both first order off-momentum $\beta$-beats and second order chromaticities are related to the half-integer resonance driving terms (RDTs). Based on the contributions from each sextupole family to the half-integer RDTs, we propose a method with 4-knobs corresponding to 4-pairs of chromatic sextupole families to correct online the second order chromaticities. We carried out numerical simulations to verify this method. From them we show that the 4-knob method does reduce the unbalance in the correction strengths among the sextupole families, and avoids the reversal of sextupole polarities. Therefore, this method yields larger dynamic apertures for the proposed RHIC $2009100 \mathrm{GeV}$ pp run lattices.

\section{Optics parameters at chromatic sextupoles}

In this section we discuss the optics parameters at all chromatic sextupoles in the RHIC adopting the proposed Blue ring lattice for the 2009 RHIC $100 \mathrm{GeV}$ pp run. For this calculation, the $\beta^{*}$ functions at IP6 and IP 8 are set to $0.7 \mathrm{~m}$, the $\beta$ functions at the other RHIC symmetric points IP10, IP12, IP2, and IP4 are set to $5 \mathrm{~m}$. The proton beams from the Blue and Yellow rings collide at IP6 and IP8. The working point without collision is set to $(28.685,29.695)$. We corrected the first order chromaticities to 1 unit with the 2 -family scheme. With this scheme, all SF sextupoles and all SD sextupoles have the same strengths.

There are 11 FODO cells in each arc. The SF and SD chromatic sextupoles are located next to the focusing and the defocusing main quadrupoles, respectively. Fig. 1 shows the horizontal dispersions at all 


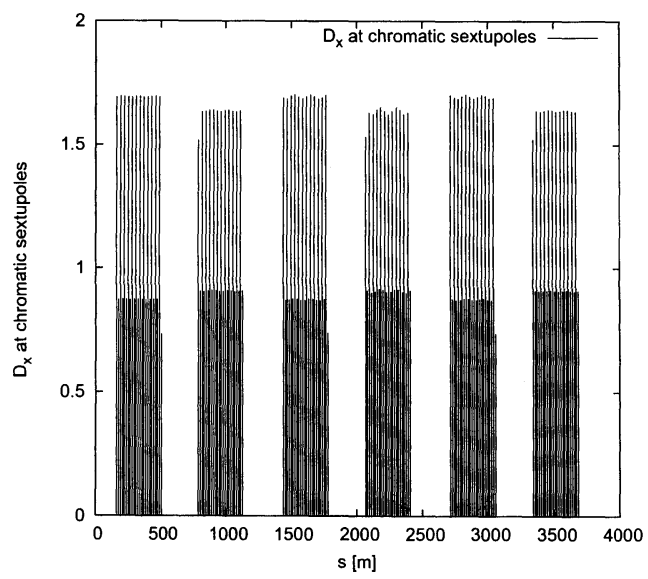

Figure 1: Horizontal dispersion $D_{x}$ at all RHIC chromatic sextupoles.
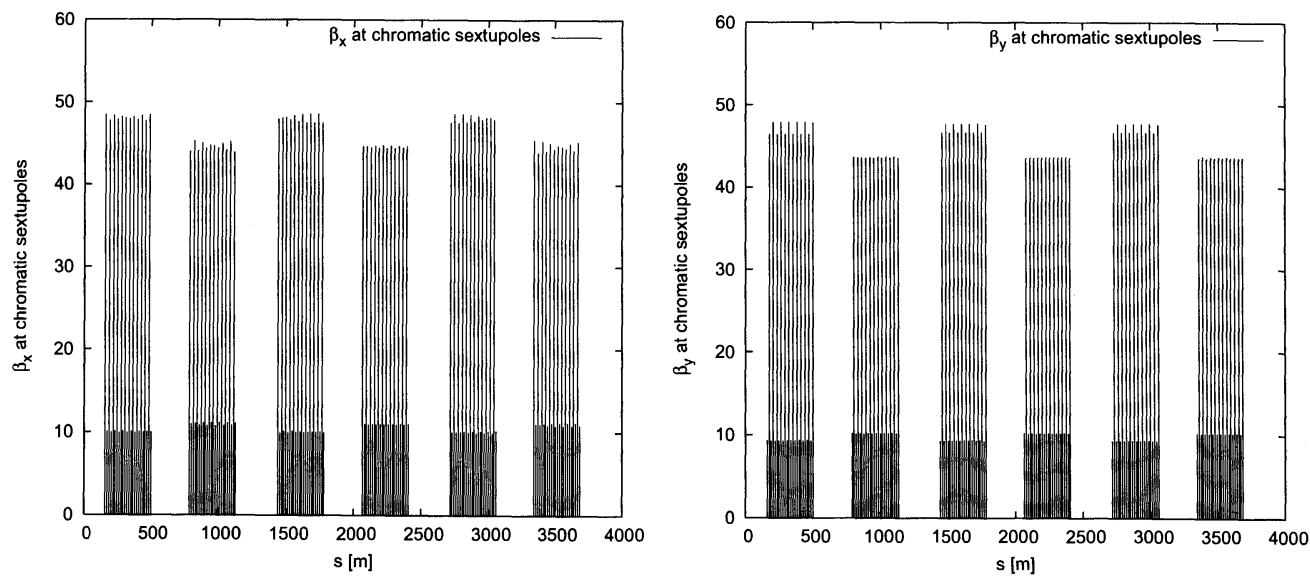

Figure 2: Left plot: $\beta_{x}$ at all RHIC chromatic sextupoles; Right plot: $\beta_{y}$ at all RHIC chromatic sextupoles.

chromatic sextupoles. The horizontal dispersion at the SF and SD sextupoles are respectively about $1.7 \mathrm{~m}$ and $0.9 \mathrm{~m}$. Fig. 2 shows the $\beta$ functions at all chromatic sextupoles. The $\beta_{x, y}$ functions at the SF and SD sextupoles are about $(48.0 \mathrm{~m}, 9.5 \mathrm{~m})$ and $(10.1 \mathrm{~m}, 48.0 \mathrm{~m})$ respectively. Fig. 3 plots the betatron phase advances at all chromatic sextupoles with respect to IP6. The horizontal and vertical phase advances of each FODO cell in the arcs are about $83^{\circ}$ and $88^{\circ}$, respectively. From Fig. 1 and Fig. 2, the dispersion and $\beta$ functions are repeated closely at all SF or all SD sextupoles.

\section{Perturbation theory}

Here we show that the first order off-momentum $\beta$-beat and second order chromaticities both are closely related to the half-integer resonance driving terms.

\subsection{First order chromaticities}

From the first order perturbation theory, the horizontal and vertical tune shifts $\Delta Q_{x, y}$ from small gradient errors in the ring are given by

$$
\Delta Q_{x, y}=\frac{1}{4 \pi} \oint \beta_{x, y}(s) \Delta k_{1}(s) d s .
$$

Here $\beta_{x, y}(s)$ are the unperturbed $\beta$ functions at location $\mathrm{s}$, and $\Delta k_{1}(s)$ is the gradient error at location s. The integral is performed all along the ring. For a particle with a relative momentum deviation $\delta$,

$$
\Delta k_{1}(s)=\left[\mp K_{1}(s) \pm K_{2}(s) D_{x}(s)\right] \delta,
$$



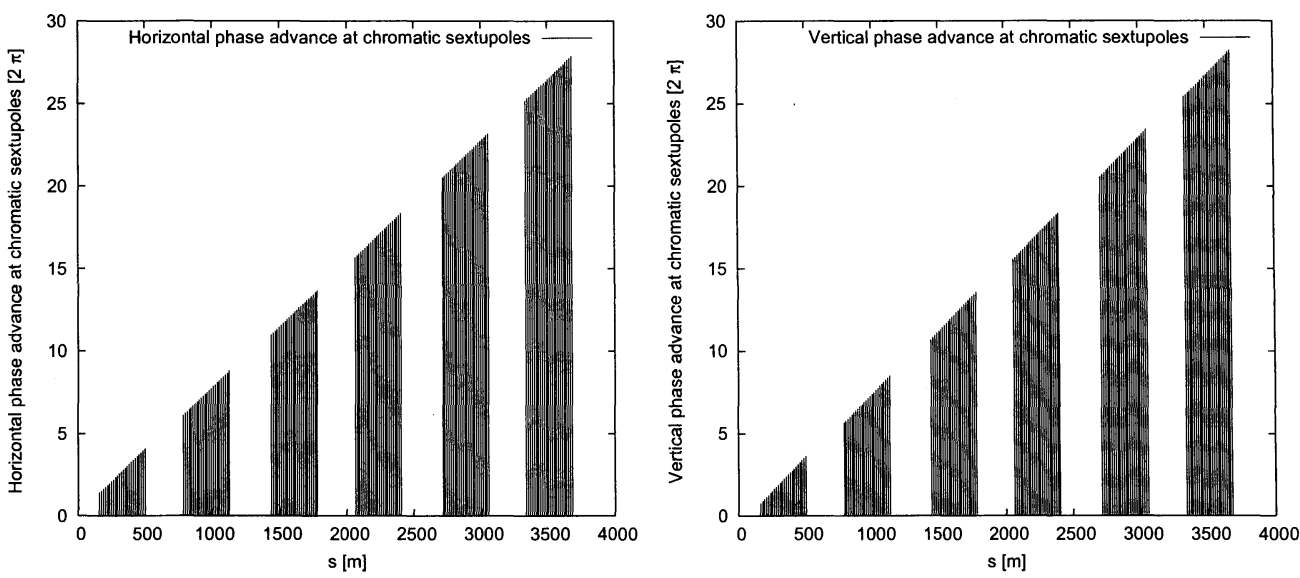

Figure 3: Left plot: Horizontal phase advances at all RHIC chromatic sextupoles; Right plot: Vertical phase advances at all RHIC chromatic sextupoles.

where $K_{n}=\left.\frac{1}{(B \rho)} \frac{\partial B_{y}^{n}}{\partial x^{n}}\right|_{x=y=0}$, and $K_{1,2}$ are the quadrupole and sextupole strengths respectively. $D_{x}(s)$ is the horizontal dispersion at location s. Integrating Eq. (2) into Eq. (1), we obtain the first order chromaticities $\xi_{x, y}^{(1)}$

$$
\xi_{x, y}^{(1)}=\frac{\partial Q_{x, y}}{\partial \delta}=\frac{1}{4 \pi} \oint \beta_{x, y}(s)\left[\mp K_{1}(s) \pm K_{2}(s) D_{x}(s)\right] d s
$$

\subsection{First order $\beta$-beat}

Also following the first order perturbation theory, the changes in $\beta$ functions due to small gradient errors are given by

$$
\frac{\Delta \beta_{x, y}(s)}{\beta_{x, y}(s)}= \pm \frac{1}{2 \sin \left(2 \pi Q_{x, y}\right)} \oint \beta_{x, y}\left(s^{\prime}\right) \Delta k_{1}\left(s^{\prime}\right) \cos \left(2\left|\phi_{x, y}(s)-\phi_{x, y}\left(s^{\prime}\right)\right|-2 \pi Q_{x, y}\right) d s^{\prime} .
$$

Plugging Eq. (2) into Eq. (4), we obtain the first order off-momentum $\beta$-beat

$$
\begin{aligned}
& \frac{1}{\beta_{x, y}(s)} \frac{\partial \beta_{x, y}(s)}{\partial \delta}= \\
& \pm \frac{1}{2 \sin \left(2 \pi Q_{x, y}\right)} \oint \beta_{x, y}\left(s^{\prime}\right)\left[\mp K_{1}\left(s^{\prime}\right) \pm K_{2}\left(s^{\prime}\right) D_{x}\left(s^{\prime}\right)\right] \cos \left(2\left|\phi_{x, y}(s)-\phi_{x, y}\left(s^{\prime}\right)\right|-2 \pi Q_{x, y}\right) d s^{\prime} .
\end{aligned}
$$

\subsection{Second order chromaticities}

The second order chromaticities can be calculated following [3]

$$
\xi_{x, y}^{(2)}=\left.\frac{1}{2} \frac{\partial^{2} Q_{x, y}}{\partial \delta^{2}}\right|_{\delta=0}=\left.\frac{1}{2} \frac{\partial \xi_{x, y}^{(1)}}{\partial \delta}\right|_{\delta=0}
$$

Plugging Eq. (3) into Eq. (6), and considering the changes of magnetic strengths, $\beta$ functions and dispersion due to the relative momentum deviation $\delta$, we obtain

$$
\xi_{x, y}^{(2)}=-\frac{1}{2} \xi_{x, y}^{(1)}+\frac{1}{4 \pi} \oint\left[\mp K_{1} \pm K_{2} D_{x}\right] \frac{\partial \beta_{x, y}}{\partial \delta} d s \pm \frac{1}{4 \pi} \oint K_{2} \beta_{x, y} D_{x}^{(2)} d s .
$$

Here, $\frac{\partial \beta_{x, y, \delta}}{\partial \delta}$ is the off-momentum $\beta$-beat given by Eq. (5), $D_{x}^{(2)}$ is the second order horizontal dispersion function, and $D_{x}^{(2)}=\left.\frac{\partial D_{x, \delta}}{\partial \delta}\right|_{\delta=0}$.

The first term in Eq. (7) comes from the changes of magnetic strengths for off-momentum particles, which is smaller than the other terms and can be ignored for most of the time. The second term in Eq. (7) arises from the first order off-momentum $\beta$-beat. The third term of Eq. (7) comes from the second order dispersion. 


\subsection{Half-integer RDTs}

From Eq. (4), the first order off-momentum $\beta$-beat is determined by the half-integer RDTs. It will be enhanced when the unperturbed tunes are close to half integer resonance. The horizontal half-integer RDT is defined as

$$
h_{20001}=\sum_{i}^{N}\left[-\left(K_{1} L\right)_{i}+\left(K_{2} D_{x} L\right)_{i}\right] \beta_{x, i} e^{-i 2 \phi_{x, i}} .
$$

The vertical half-integer RDT is defined as

$$
h_{00201}=\sum_{i}^{N}\left[+\left(K_{1} L\right)_{i}-\left(K_{2} D_{x} L\right)_{i}\right] \beta_{y, i} e^{-i 2 \phi_{y, i}} .
$$

In Eq. (8) and (9) we used summations rather than integrals. The half-integer RDTs $h_{20001}$ and $h_{00201}$ are defined at the starting point of the lattice. In our following discussion, the starting point of the lattice is IP6.

Half-integer RDTs $h_{20001}$ and $h_{00201}$ will cause $2 Q_{x}$ and $2 Q_{y}$ betatron resonances respectively. From Eq. (2) and Eq. (7), the first order off-momentum $\beta$-beats and the second order chromaticities both are closely related to the half-integer RDTs $h_{20001}$ and $h_{00201}$.

\section{Calculating half-integer RDTs}

In this section we will calculate the contributions to the half-integer RDTs from the chromatic sextupoles and sort them into several families for easy and effective half-integer RDT minimization. In the calculations, we assumed that the integrated strengths of individual sextupoles are $\left(K_{2} L\right)=1 \mathrm{~m}^{-2}$. The residual halfinteger RDTs from the quadrupole magnets and the sextupole field errors in the interaction regions are not calculated. Considering that the half-integer RDTs are complex, in the following we show both the real and imaginary parts of the horizontal and vertical half-integer RDTs for each case. Further, we use the Blue ring lattice proposed for 2009 RHIC $100 \mathrm{GeV}$ polarized proton run.

\subsection{Contributions from the SF sextupoles in the first arc}

As an example, we first check the contributions from the focusing chromatic sextupoles in the first outer arc from IP6 to IP8. The contributions from the defocusing chromatic sextupoles in this arc, or the focusing or defocusing chromatic sextupoles in other arcs can be calculated in the same way.

The SF sextupoles between IP6 and IP8 were sorted as $6^{*}(\mathrm{SFPO}, \mathrm{SFMO})$ in the RHIC control system. Fig. 4 shows the contributions of the 12 focusing chromatic sextupoles to the horizontal and vertical halfinteger RDTs. The left plot of Fig. 4 shows their contributions to the horizontal RDT, and the right plot of Fig. 4 shows their contributions to the vertical RDT.

From Fig. 4, according to their contributions to the half-integer RDTs, the 12 focusing chromatic sextupoles in the first arc clearly can be sorted into two groups, that is, SFPO and SFMO. The contributions from the 6 individual sextupoles in each group of SFPO or SFMO have similar contribution amplitudes and angles to the half-integer RDTs.

Also from Fig. 4, we note that the contributions to the horizontal or the vertical half-integer RDT from the SFPO and SFDO sextupoles in this arc are almost 180 degree apart. Therefore, if the SFPO and SFDO are powered with the same strengths, their contributions to the half-integer RDTs will cancel. However, if the SFPO and SFDO are powered with opposite strengths, they will give net contributions to the half-integer RDTs.

\subsection{Contributions from 24 sub-families}

There are 24 independent power supplies to power all the 144 chromatic sextupoles in each RHIC ring. Here we evaluate the contributions from all of the 24 sub-families to the half-integer RDTs. In the RHIC control system, the chromatic sextupoles in all 3 outer arcs have the same powering pattern $6^{*}$ (SFPO, SDMO, SFMO, SDMO), while the chromatic sextupoles in all 3 inner arcs have the same powering pattern $6^{*}$ (SFPI, SDMI, SFMI, SDMI). Here we don't change the names of the 24 sub-families, but we note that there are 3 sub-families ( or 3 independent power supplies ) for each family of SFPO, SDMO, SFMO, SDMO,SFPI, SDMI, SFMI and SDMI . 

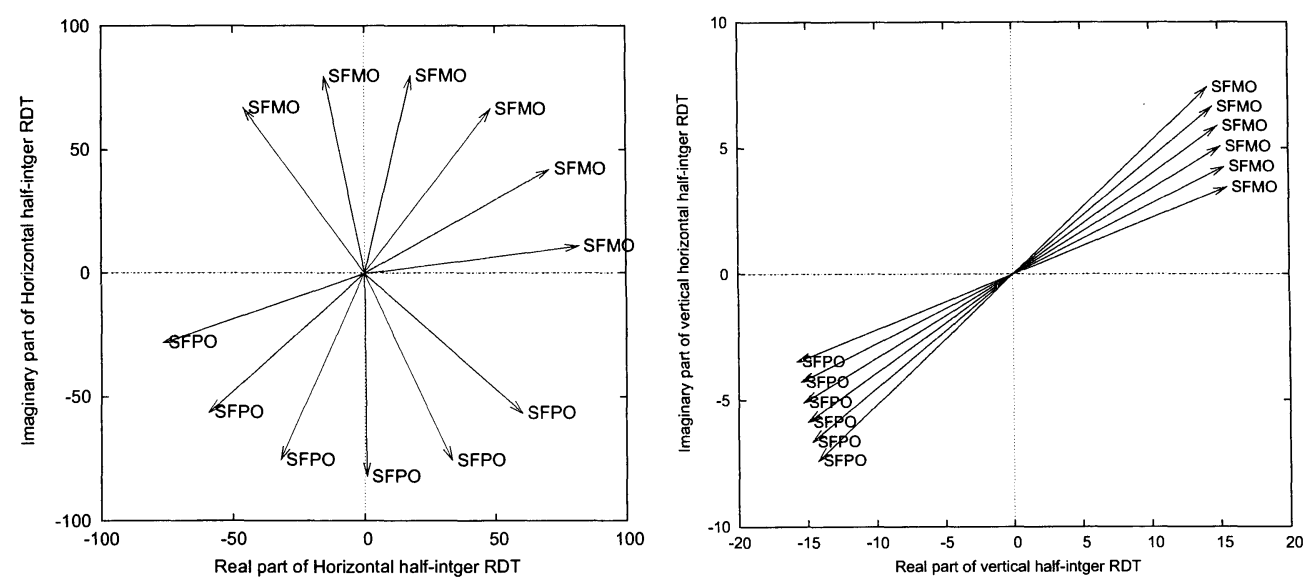

Figure 4: Left plot: Contributions to the horizontal half-integer RDT from all SFs in first RHIC Blue arc. Right plot: Contributions to the vertical half-integer RDT from all SFs in first RHIC Blue arc.

Fig. 5 shows the contributions of all the SF sub-families to the half-integer RDTs. Fig. 6 shows the contributions of all SD sub-families to the half-integer RDTs. In Fig. 5 and Fig. 6, the left side plot shows their contributions to the horizontal half-integer RDT and the right side plot shows their contributions to the vertical half-integer RDT.

From Fig. 5 and Fig. 6, the SF sub-families have larger contributions to the horizontal half-integer RDT and have smaller contributions to the vertical half-integer RDT. The SD sub-families have smaller contributions to the horizontal half-integer RDT and have larger contributions to the vertical half-integer RDT.

Fig. 5 and Fig. 6 also show that there are partial cancellations in the 3 sub-families among each family of SFPO, SFMO, SFPI, SFMI, SDPO, SDMO, SDPI, and SDMI. This is caused by the 3 -folding symmetric RHIC ring lattice and the ring working point $(28.685,29.695)$. For example, the contribution angles to the horizontal half-integer RDT from the same name sextupole sub-families in the adjacent outer or inner arcs are separated by $28.685 \times 2 \pi \times 2 / 3=38.2467 \pi$, or about $45^{\circ}$.

\subsection{Contributions from 8 families}

In the RHIC control system, the above 24 sub-families haves been further broken down into 8 families. All the outer or the inner arcs have the same chromatic sextupole powering patterns. Ignoring the cancellations in each family, Fig. 7 shows the contributions from the 8 sextupole families to the half-integer RDTs. The left plot shows their contributions to the horizontal half-integer RDT and the right plot shows their contributions to the vertical half-integer RDT.

Previously, we used 8 families or 8 variables to minimize the second and the third order chromaticities and the first order off-momentum $\beta$-beats at IP6 with the Harmon module in the MAD8 $[4,5,6]$. Harmon uses methods of general multi-dimensional nonlinear optimization to reduce the overall error function $\chi^{2}$. Most of the time, the Harmon module gives very good corrections and the second order chromaticities normally are less than 50 units after corrections. However, we notice that the Harmon module some time fails to avoid the reversal of polarity of sextupole families, and introduces a large unbalance in the correction strengths among the 8 families for the low $\beta^{*}$ RHIC 2009 run pp lattices.

\subsection{4 knobs to minimize half-integer RDTs}

From Fig. 8, we clearly see the opposite contributions to the half-integer RDTs from the 2 families in each pairs (SFPO and SFMO), (SDPO and SDMO), (SFPI and SFMI), and (SDPI and SDMI). For example, for the pair of (SFPO and SFMO), if the SFPO and SFMO families are powered with the same strength their contributions to the half-integer RDTs will cancel. However, if the SFPO and SFMO families are powered oppositely with the same absolute strength, they will give net contributions to the half-integer RDTs.

As seen in Fig. 8, the two pairs (SFPO and SFMO) and (SFPI and SFMI) have larger contributions to the horizontal half-integer RDT, while the two pairs (SDPO and SDMO) and (SDPI and SDMI) have larger contributions to the vertical half-integer RDT. Therefore, adjusting the pair of (SFPO and SFMO) 

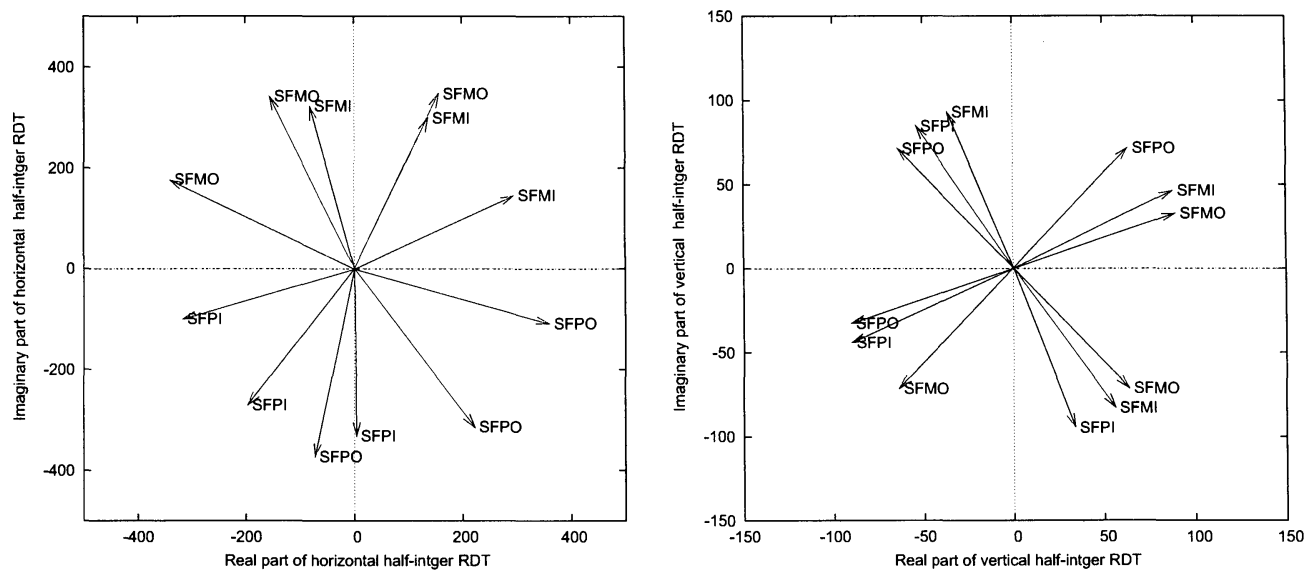

Figure 5: Contributions of all SF sextupole families to the horizontal and vertical half-integer RDTs. Left plot: Contributions to the horizontal half-integer RDT; Right plot: Contributions to the vertical half-integer RDT.
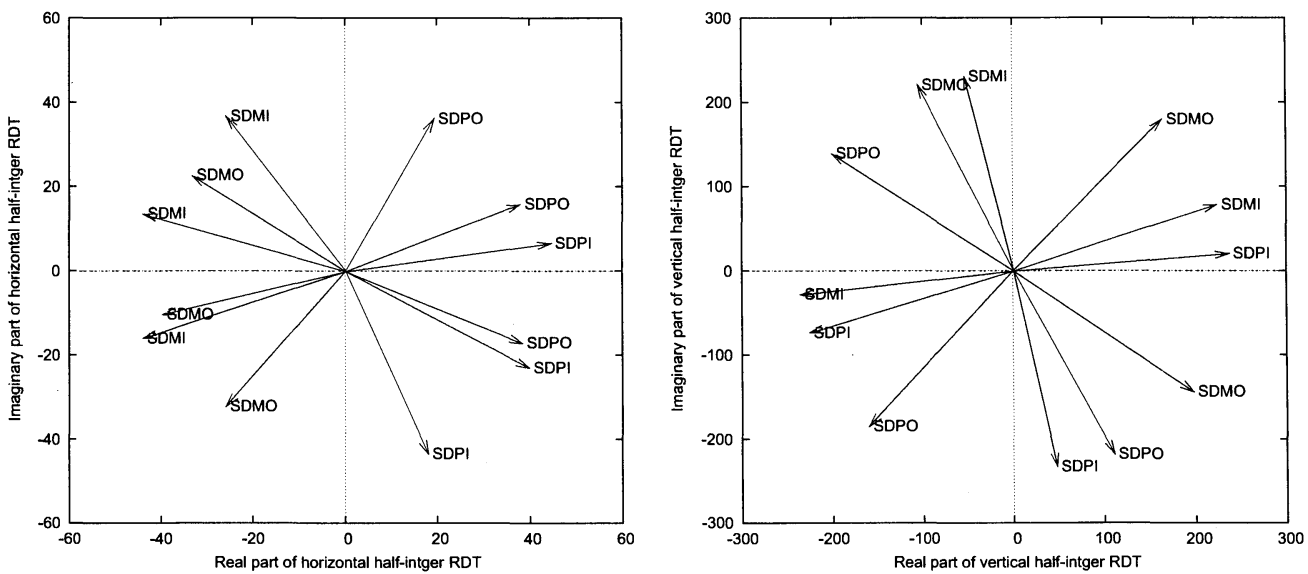

Figure 6: Contributions of all SD sextupole families to the half-integer RDTs. Left plot: Contributions to the horizontal half-integer RDT; Right plot: Contributions to the vertical half-integer RDT.
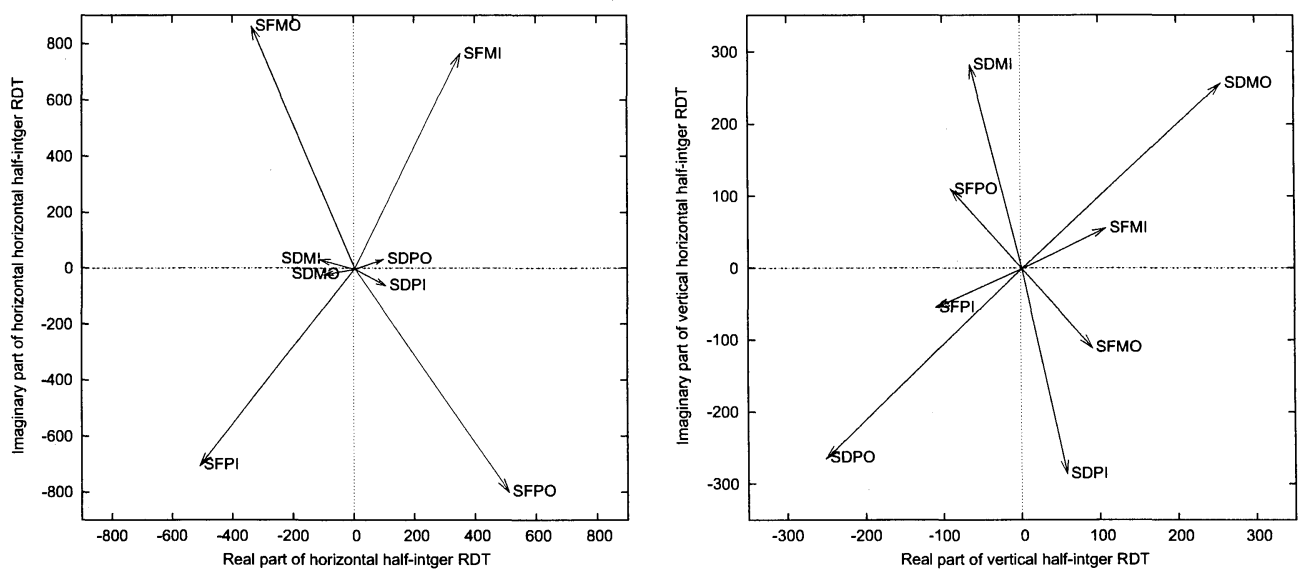

Figure 7: Contributions of all 8 sextupole families to the half-integer RDTs. Left plot: Contributions to the horizontal half-integer RDT; Right plot: Contributions to the vertical half-integer RDT. 
or of (SFPI and SFMI) mostly will affect the the horizontal second order chromaticity and the first order horizontal off-momentum $\beta$-beat, while adjusting the pair of (SDPO and SDMO) or of (SDPI and SDMI) mostly will affect the vertical second order chromaticity and the vertical first order vertical off-momentum $\beta$-beat.

Fig. 8 also reveals that the contributions from the 2 pairs of (SFPO and SFMO) and (SFPI and SFMI), or from the 2 pairs of (SDPO and SDMO) and (SDPI and SDMI) are not orthogonal. This is caused by the 3 -folding symmetric RHIC ring lattice and the working point.

In summary, there are 4 knobs corresponding to 4 pairs of chromatic sextupole families to easily and effectively minimize half-integer RDTs to correct the second order chromaticities in the RHIC. The 4 knobs are the 4 pairs of chromatic sextupole families (SFPO and SFMO), (SFPI and SFMI) (SDPO and SDMO), and (SDPI and SDMI). In each correction step, we adjust the two families of one pair with same amount of absolute strength but with opposite signs.

This method was tested in the 2007 RHIC beam experiment session. It is now being implemented in the RHIC online control system. The 4-knob method is easy and effective to correct the second order chromaticities on line where the residual half-integer RDTs normally are unknown. In each step of knobbing, the second order chromaticities can be used as the criterion to determine the effects of the correction strengths that we put in. The second order chromaticities are determined by a tune measurement after shifting the $\mathrm{RF}$ frequency in the RHIC. Another advantage of the 4-knob method is that it does not change the first order chromaticities during the corrections of second order chromaticities.

\section{Offline simulation of $\xi_{x, y}^{(2)}$ correction}

In this section, we numerically simulate the second order chromaticity correction with the above 4-knob method, using the Blue ring lattice proposed for 2009 RHIC $100 \mathrm{GeV}$ polarized proton run. Before correction, the first order chromaticities are corrected with the 2 -family scheme to 1 unit. Table 1 shows the chromaticities and correction strengths with this 2 -family correction scheme. The unit of the sextupole strengths is $\mathrm{m}^{-3}$.

On top of the 2-family scheme correction, we use the above 4 knobs to adjust the second order chromaticities. In each step, we knob the two families of one pair with a same amount of absolute strength but with opposite signs, that is, we increase the strengths of one family and decrease the strength of another family by the same amount. The second order chromaticities are used as the criterion to determine the effect of the correction strengths we put in.

With the proposed RHIC run09 $100 \mathrm{GeV}$ polarized proton lattice with $\beta^{*}=0.7 \mathrm{~m}$, we find that the pair (SFPI and SFMI) will significantly affect the horizontal second order chromatiicty while the pair (SDPO and SDMO) will significantly affect the vertical second order chromaticity. After a few of iterations, we easily find the correction strengths of the 8 sextupole families to reduce the second order chromaticities to below 500. Fig. 9 shows the off-momentum tunes before and after such correction with the 4 -knob method. Fig. 10 shows the off-momentum $\beta$-beat in the first $200 \mathrm{~m}$ from IP6 before and after the second order chromaticity correction. From Fig. 10, we note that the first order off-momentum $\beta$-beats also are reduced after the second order chromaticities are corrected.

Table 2 shows the required sextupole correction strengths from the Harmon module and from the 4-knob method. The unit of the sextupole strengths is $\mathrm{m}^{-3}$. Three proposed Blue ring lattices with $\beta^{*}=0.9 \mathrm{~m}$, $0.7 \mathrm{~m}, 0.5 \mathrm{~m}$ for the RHIC $2009100 \mathrm{GeV}$ polarized proton run are used. From Table 2, the Harmon module gives much smaller second order chromaticties than does the 4-knob method. With the latter, we normally stop correction iterations after the second order chromaticities are below 500. For the RHIC operation $\xi_{x, y}^{(2)}$ below 500 are acceptable.

Table 2 shows that the correction strengths from the Harmon module are bigger than that from 4-knob method. The correction strengths from the Harmon module have a big unbalance among the 8 sextupoles families. For example, for the $\beta^{*}=0.5 \mathrm{~m}$ lattice, the biggest and the smallest required correction strengths of sextupole families from the Harmon correction respectively are $1.96 \mathrm{~m}^{-3}$ and $0.08 \mathrm{~m}^{-3}$. While with the 4-knob method, the biggest and the smallest required correction strengths are $1.12 \mathrm{~m}^{-3}$ and $0.31 \mathrm{~m}^{-3}$. As seen in Table 2, SDPO is required to change polarity with Harmon optimization. However, all power supplies of RHIC chromatic sextupoles are monopoles.

Table 3 shows the calculated $10^{6}$ turn dynamic apertures with the second order chromaticity corrections with the Harmon module and the 4 -knob method. The non-collisional working point is $(28.685,29.695)$. The bunch intensity is set to $1.8 \times 10^{11}$ and the normalized $95 \%$ beam emittance is $20 \pi \mathrm{mm}$.mrad. Comparison 


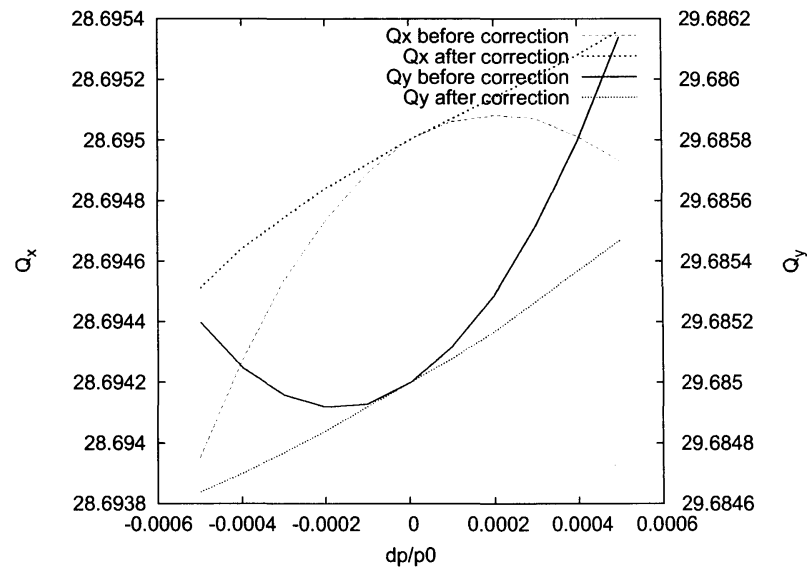

Figure 8: Off-momentum tunes before and after $\xi^{(2)}$ correction.

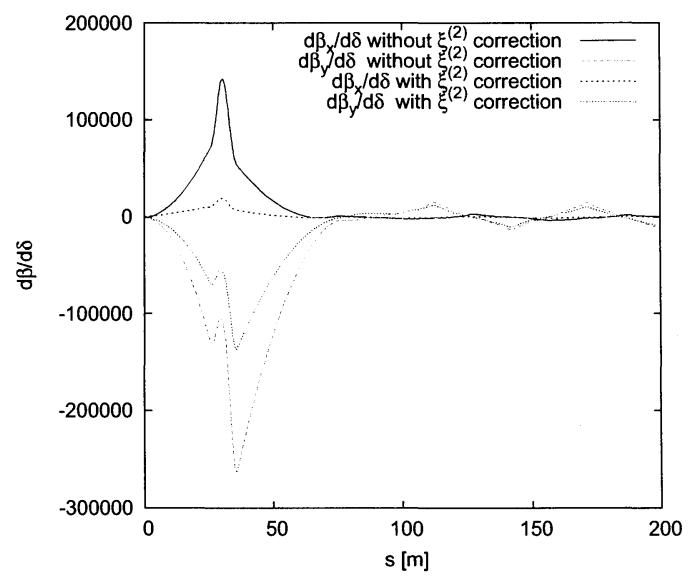

Figure 9: Off-momentum $d \beta / d \delta$ before and after $\xi^{(2)}$ correction.

reveals that the correction strengths from the 4-knob method gives higher dynamic apertures than those from the Harmon module for all three lattices with $\beta^{*}=0.9 \mathrm{~m}, 0.7 \mathrm{~m}, .0 .5 \mathrm{~m}$.

Thus, although the 4-knob method does not correct the second order chromaticities to values as close to zero as the Harmon module, it does lessen the unbalance in the correction strengths among the sextupole families, and avoids reversing the polarities of the sextupoles. Therefore, it yields larger dynamic apertures than that with the Harmon correction strengths. The 4-knob method is readily implemented and used for the online second order chromaticities in the RHIC polarized proton run even though the online lattice model does not reproduce the actual one well.

Table 1: Chromaticities and sextupole correction strengths with the 2-family scheme.

\begin{tabular}{lccc}
\hline \hline & \multicolumn{3}{c}{ Lattices } \\
Quantities & $\beta^{*}=0.9 \mathrm{~m}$ & $\beta^{*}=0.7 \mathrm{~m}$ & $\beta^{*}=0.5 \mathrm{~m}$ \\
\hline$\xi_{x, y}^{(1)}$ & $(1,1)$ & $(1,1)$ & $(1,1)$ \\
$\xi_{x, y}^{(2)}$ & $(-2538,2806)$ & $(-3072,3963)$ & $(-3739,6374)$ \\
$K_{2}$ for all SFs & 0.31 & 0.36 & 0.42 \\
$K_{2}$ for all SDs & -0.60 & -0.68 & -0.80 \\
\hline \hline
\end{tabular}


Table 2: Required sextupole correction strengths from Harmon module and from 4-knob method.

\begin{tabular}{lcc}
\hline \hline Sextupole Families & $\begin{array}{c}\text { Correction strengths } \\
\text { from Harmon }\end{array}$ & $\begin{array}{c}\text { Correction strengths } \\
\text { from 4-knobs }\end{array}$ \\
\hline$\beta^{*}=0.9$ m lattice: & 0.47 & 0.31 \\
SFPO & 0.40 & 0.31 \\
SFMO & 0.31 & 0.39 \\
SFPI & 0.07 & 0.23 \\
SFMI & -0.21 & -0.41 \\
SDPO & -0.74 & -0.78 \\
SDMO & -0.56 & -0.60 \\
SDPI & -0.88 & -0.60 \\
SDMI & $(44,10)$ & $(-449,435)$ \\
corrected $\xi_{x, y}^{(2)}$ & & \\
\hline$\beta^{*}=0.7$ m lattice: & 0.55 & 0.36 \\
SFPO & 0.50 & 0.36 \\
SFMO & 0.33 & 0.46 \\
SFPI & 0.05 & 0.25 \\
SFMI & -0.07 & -0.44 \\
SDPO & -0.76 & -0.92 \\
SDMO & -0.75 & -0.68 \\
SDPI & -1.14 & -0.68 \\
SDMI & $(32,-16)$ & $(330,464)$ \\
corrected $\xi_{x, y}^{(2)}$ & & \\
\hline$\beta^{*}=0.5$ m lattice: & 0.58 & 0.42 \\
SFPO & 0.62 & 0.42 \\
SFMO & 0.40 & 0.53 \\
SFPI & 0.08 & 0.31 \\
SFMI & 0.67 & -0.48 \\
SDPO & -0.31 & -1.12 \\
SDMO & -1.59 & -0.80 \\
SDPI & -1.96 & -0.80 \\
SDMI & $(-91,-22)$ & $(341,413)$ \\
corrected $\xi_{x, y}^{(2)}$ & & \\
\hline \hline & & \\
\hline
\end{tabular}

Table 3: Dynamic apertures in $10^{6}$ turn trackings

\begin{tabular}{lcccccc}
\hline \hline Case & $15^{\circ}$ & $30^{\circ}$ & $45^{\circ}$ & $60^{\circ}$ & $75^{\circ}$ & $\mathrm{DA}_{\min }$ \\
\hline$\beta^{*}=0.9 \mathrm{~m}$ lattice: & & & & & & \\
no $\xi^{(2)}$ corr & 5.7 & 5.8 & 5.4 & 4.9 & 5.4 & 4.9 \\
$\xi^{(2)}$ corr with Harmon & 6.1 & 5.9 & 5.6 & 3.6 & 4.5 & 3.6 \\
$\xi^{(2)}$ corr with 4-knobs & 5.8 & 5.8 & 5.4 & 4.9 & 4.5 & 4.5 \\
\hline$\beta^{*}=0.7 \mathrm{~m}$ lattice: & & & & & & \\
no $\xi^{(2)}$ corr & 4.7 & 4.7 & 4.5 & 4.1 & 4.2 & 4.1 \\
$\xi^{(2)}$ corr with Harmon & 4.9 & 4.9 & 4.7 & 3.9 & 3.5 & 3.5 \\
$\xi^{(2)}$ corr with 4-knobs & 5.1 & 5.2 & 4.5 & 4.2 & 4.1 & 4.1 \\
\hline no $\xi^{(2)}$ corr & 3.9 & 3.6 & 3.4 & 3.0 & 2.9 & 2.9 \\
$\xi^{(2)}$ corr with Harmon & 3.9 & 4.1 & 3.6 & 3.4 & 4.3 & 3.4 \\
$\xi^{(2)}$ corr with 4-knobs & 4.4 & 4.3 & 3.9 & 3.6 & 3.5 & 3.5 \\
\hline \hline
\end{tabular}




\section{Conclusion}

Based on the contributions of the chromatic sextupole families to the half-integer resonance driving terms in the RHIC rings, we sorted the RHIC chromatic sextupoles into 4 pairs or 4 knobs to correct online the second order chromaticties. The 4-knob method was tested in the 2007 RHIC beam experiment session and is being implemented in the RHIC online control system. The method will not change the first order chromaticities during knobbing. It works even if the online RHIC optics model does not closely reproduce the real machine. From our numeric simulations, we verified that the 4 -knob method reduces the unbalance in the correction strengths among the sextupole families, and avoids reversing sextupole polarities. Accordingly, it yields larger dynamic apertures for the Blue ring lattices proposed for the RHIC $2009100 \mathrm{GeV}$ polarized proton run.

\section{Acknowledgments}

We thank M. Bai, J. Beebe-Wang, C. Montag, T. Roser, and T. Satogatafor stimulating discussions and thank A. Woodhead for the help in the preparing this manuscript. This work is supported by U.S. DOE under contract No. DE-AC02-98CH10886.

\section{References}

[1] W. Fischer, Beam-beam and BTF, 2006 RHIC Accelerator Physics Experiments Workshop, November 2-3, 2005, BNL. Available at http://www.c-ad.bnl.gov/APEX/APEXWorkshop2006/agenda.htm.

[2] S. Tepikian, Nonlinear chromaticity correction, 2006 RHIC Retreat, July 10-13, 2006, Port Jefferson, NY. Available at http://www.c-ad.bnl.gov/RHIC/Retreat2006/agenda.html.

[3] J. Bengtsson, The sextupole scheme for the Swiss Light Source(SLS): an analytical approach, SLS Note 9/97, March 7, 1997.

[4] MAD8 code homepage, http://mad.home.cern.ch/mad/.

[5] Y. Luo, Online nonlinear chromaticity correction at RHIC store, November 2-3, 2006, RHIC 2006 Accelerator Physics Experiment Workshop, November 2-3, 2005, BNL, Upton, NY. Available at http://www.c-ad.bnl.gov/APEX/APEXWorkshop2006/agenda.htm.

[6] Y. Luo, Nonlinear chromaticity correction in RHIC run 2007, 2007 RHIC Retreat, July 16-17, 2007, Foxwood, CT. Available at http://www.c-ad.bnl.gov/RHIC/retreat2007/. 